\title{
Primary Synovial Diffuse Large B-Cell Lymphoma Presenting as Loosening of Prosthetic Joint: A Case Report and Review of Literature
}

\author{
Kavita Agrawal ${ }^{\mathrm{a}, \mathrm{d}}$, Nirav Agrawal ${ }^{\mathrm{b}}$, Miles Levin ${ }^{\mathrm{c}}$
}

\begin{abstract}
Primary synovial diffuse B-cell lymphoma is a rare clinical condition. The condition presents primarily with localized joint symptoms such as swelling, pain and reduced range of motion. It may or may not be associated with lymphadenopathy, hepatosplenomegaly or B-type constitutional symptoms. We report a case of a 74-year-old woman who presented with persistent left knee pain and swelling after left knee replacement secondary to osteoarthritis. There was a concern for mechanical loosening of internal left knee prosthetic joint. On revision surgery (14 weeks after the initial surgery), hypertrophied synovium with areas of fibrotic scars, necrotic tissue and dark colored masses was resected. She was found to have diffuse large B-cell lymphoma (DLBCL) after histological analysis. In cases with persistent joint symptoms or postoperative complications, arthroscopy or arthrotomy should be considered and any atypical appearing tissue should be sent for histopathological analysis.
\end{abstract}

Keywords: Diffuse large B-cell lymphoma; Synovial; Osteoarthritis

\section{Introduction}

Non-Hodgkin's lymphoma (NHL) consists of different subtypes of neoplasms derived from B-cell or T-cell or natural killer cell lineage. B-cell lymphoma accounts for $85 \%$ of all cases of NHL. Musculoskeletal involvement can be seen in $5-25 \%$ cases of NHL either as metastasis or rarely as a primary lymphoma of bone or soft tissue [1]. The primary lymphoma of synovium is extremely rare. As a result, the diagnosis can be missed.

We report a very rare case of primary synovial diffuse

Manuscript submitted June 10, 2019, accepted July 4, 2019

aDepartment of Internal Medicine, Overlook Medical Center, Summit, NJ 07901, USA

${ }^{b}$ The Feinstein Institute for Medical Research, Manhasset, NY 11030, USA

'Department of Pathology, Overlook Medical Center, Summit, NJ 07901, USA

${ }^{\mathrm{d} C}$ Corresponding Author: Kavita Agrawal, Department of Internal Medicine, Overlook Medical Center, 99 Beauvoir Avenue, Summit, NJ 07901, USA.

Email: kaviagrawa189@gmail.com

doi: https://doi.org/10.14740/wjon1213 large B-cell lymphoma (DLBCL) presenting as a postoperative complication of mechanical loosening of internal left knee prosthetic joint. This was seen 14 weeks after a left total knee replacement was performed in a patient with long standing osteoarthritis.

\section{Case Report}

A 74-year-old woman was admitted to our hospital for elective left knee replacement for osteoarthritis. Patient had chronic left knee pain for the prior 10 years. She had been diagnosed with osteoarthritis based on the X-ray of the knee. Initially, the pain was managed with non-steroidal anti-inflammatory medications. However, over the course of past 1 year, the pain had worsened and was interfering with her daily activities. She denied any fever, chills, numbness, tingling or pain in other joints. She denied weight loss, night sweats or new lumps.

She had past medical history of hypertension and bilateral knee osteoarthritis. She had past surgical history of right knee replacement with no complications 6 months prior to this admission. She was a former smoker with history of 3 - 4 cigarettes for 20 years. She denied any alcohol or recreational drug use. Her family history included gastric cancer in the mother who died at unknown age.

Vitals were within normal range. Physical examination was normal except of left knee which revealed moderate effusion and tenderness to palpation of medial and lateral joint space. There was no erythema or warmth noted. Range of motion was mildly restricted.

X-ray of left knee showed marked degenerative changes with diffuse joint space narrowing of the medial and lateral compartments with subchondral cystic changes and degenerative spurring suggestive of moderate osteoarthritis.

\section{Treatment course}

With informed consent from the patient, the orthopedic surgeon proceeded with left total knee replacement. Intraoperatively, markedly hypertrophic synovium and areas of fibrotic tissue was noted. A large effusion was present which was clear. The fluid was sent for culture. Also, synovium and intra-articular tissue biopsies were taken and sent for culture. Aerobic, an- 


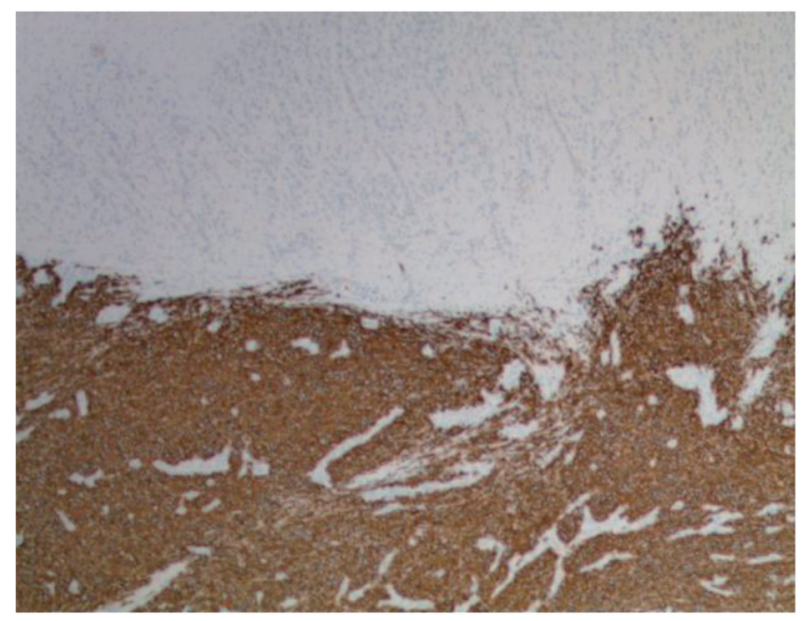

Figure 1. Low power $(\times 20)$ image showing the reactive thick fibrosynovial tissue within the knee joint, with associated giant cell reaction to cement (top of picture) with the atypical subjacent diffuse mononuclear cell infiltrate. The differential includes both reactive/infectious conditions and malignancy.

aerobic, acid fast bacilli stain and fungal culture was negative. The patient tolerated the procedure well. She was discharged on day 3 postoperatively.

Ten weeks after the first procedure, the patient followed up with orthopedics for persistent left knee pain, swelling and reduced mobility. She was recommended to undergo manipulation of left knee under anesthesia due to concern for postoperative adhesions formation. Patient agreed for the procedure. Intraoperatively, a scant amount of bloody fluid was aspirated and resent for cultures. The cultures were negative. The patient tolerated the procedure well and was discharged in 2 days postoperatively. Post-procedure, she continued to have severe left knee pain, swelling and reduced mobility. Orthopedics was concerned about mechanical loosening of internal left knee prosthetic joint. Fourteen weeks after the first procedure, she underwent revision left knee replacement surgery. Intraoperatively, she had marked arthrofibrosis of left knee. The tissue

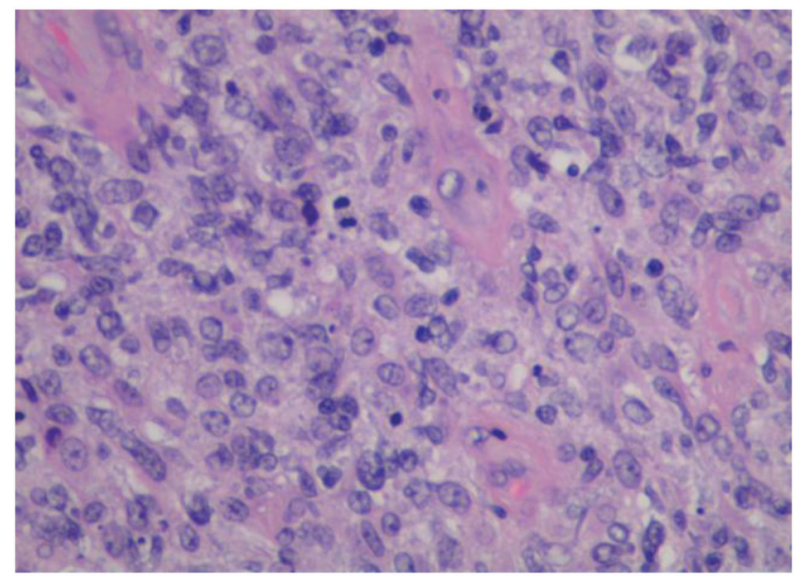

Figure 2. High power $(\times 400)$ image showing the diffuse infiltrate of large lymphoma cells with admixed histiocytes, mitosis and karyorrhexis debris.

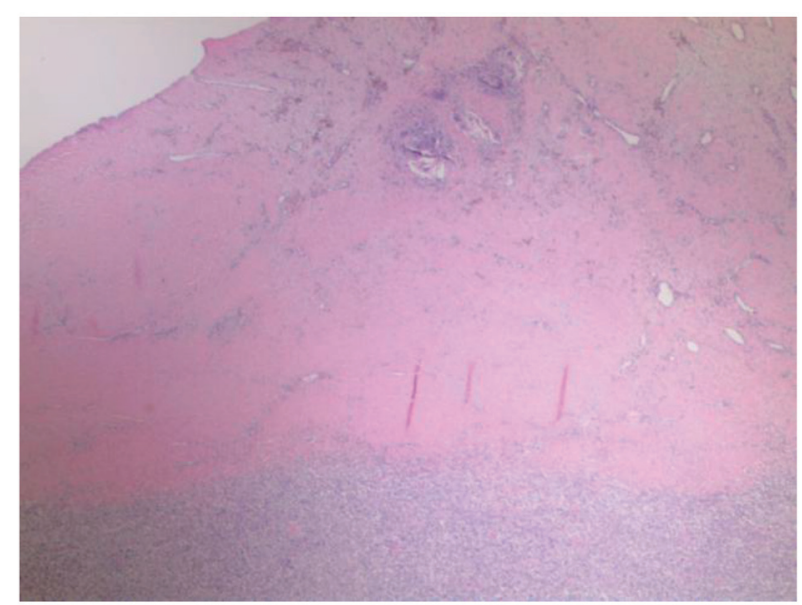

Figure 3. Low power $(\times 40)$ image of CD20 immunohistochemical stain confirming the morphologic suspicion of diffuse large B-cell lymphoma.

was hypertropied with white fibrous scars, areas of necrosis and dark colored masses. During this revision surgery, synovium and intra-articular tissue was sent for pathological analysis.

Pathological analysis of the resected tissue showed diffuse lymphoid infiltrate within fibrous tissue of medium to large atypical cells. The cells had irregular nuclei, moderate cytoplasm and conspicious nucleoli (Figs. 1 and 2). Immunohistochemistry was positive for CD20, CD10, BCL-2 and BCL6 (Fig. 3). The findings were consistent with germinal center DLBCL. Epstein-Barr virus testing was negative. The Ki-67 proliferation index averaged $60 \%$, but was up to $80 \%$ focally. Fluorescence in situ hybridization (FISH) was negative for BCL2/BCL-6/MYC rearrangements.

The patient was referred to the oncologist. Complete blood count showed white cell count of $7,350 / \mu \mathrm{L}$ with normal differential, hemoglobin $9.9 \mathrm{~g} / \mathrm{dL}$ and platelets of $375 \times 10^{3} / \mu \mathrm{L}$. Lactate dehydrogenase (LDH) was elevated to $359 \mathrm{U} / \mathrm{L}$. Antinuclear antibody (ANA) test was negative. Rheumatoid factor (RF) was negative. Positron emission tomography/computed tomography (PET/CT) from skull base to the upper thigh showed hypermetabolic activity in the region of left knee prosthesis with maximum standardized uptake value (SUV) of 25.9 consistent with lymphoma. It also showed hypermetabolic left inguinal lymphadenopathy with SUV of 12.7 and pelvic sidewall lymphadenopathy with SUV of 14.1. DLBCL was staged as II-E. She was treated with rituximab, cyclophosphamide, doxorubicin, vincristine and prednisone (R-CHOP) chemotherapy.

\section{Discussion}

DLBCL is the most common type of NHL. It accounts for approximately $25 \%$ of all NHLs [2]. It can manifest clinically with variable symptoms. The typical presentation includes nodal enlargement most commonly in neck or abdomen. But it can present as a mass lesion anywhere in the body. About $30 \%$ of the patients may have systemic B symptoms such as fever, night sweats or weight loss [3]. Extranodal DLBCL can be present in up to $40 \%$ of the cases [4]. The most common site 


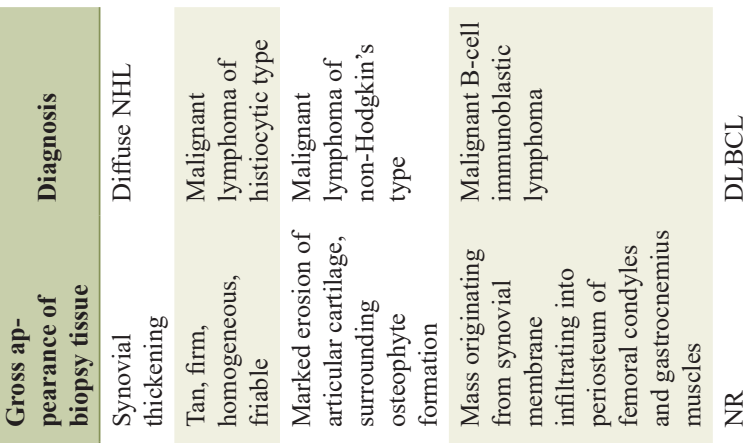
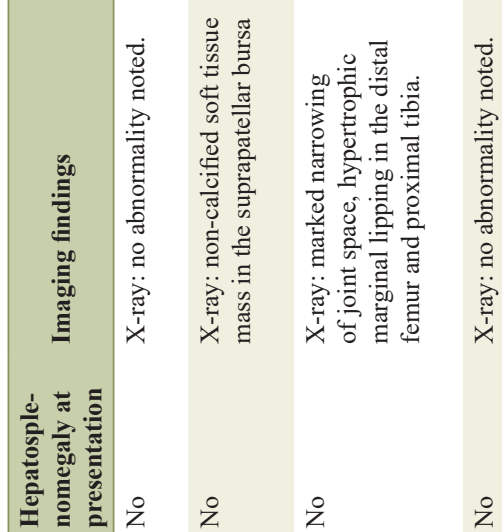

离

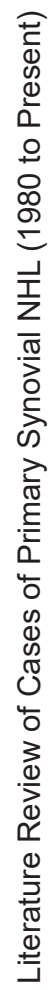

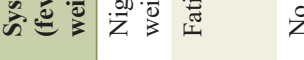

ż

ż g

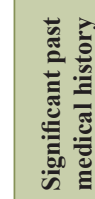

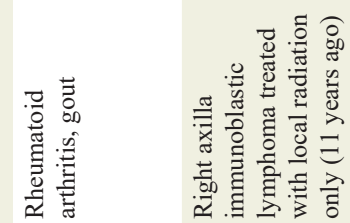

ž

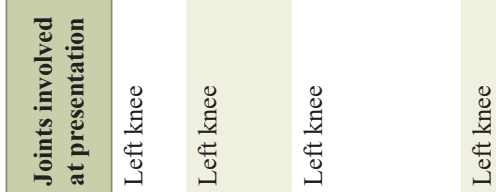

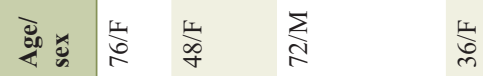

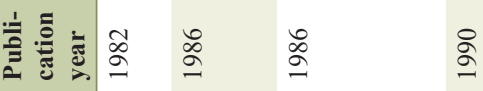

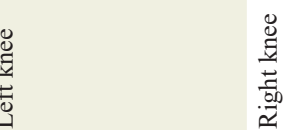

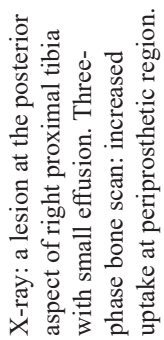

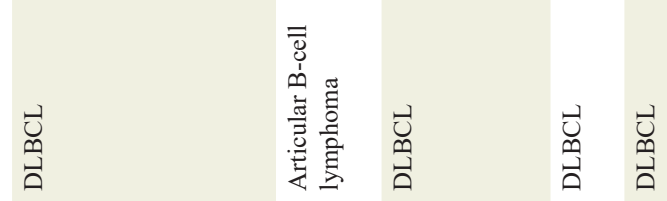

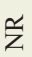

艺芓

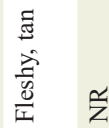
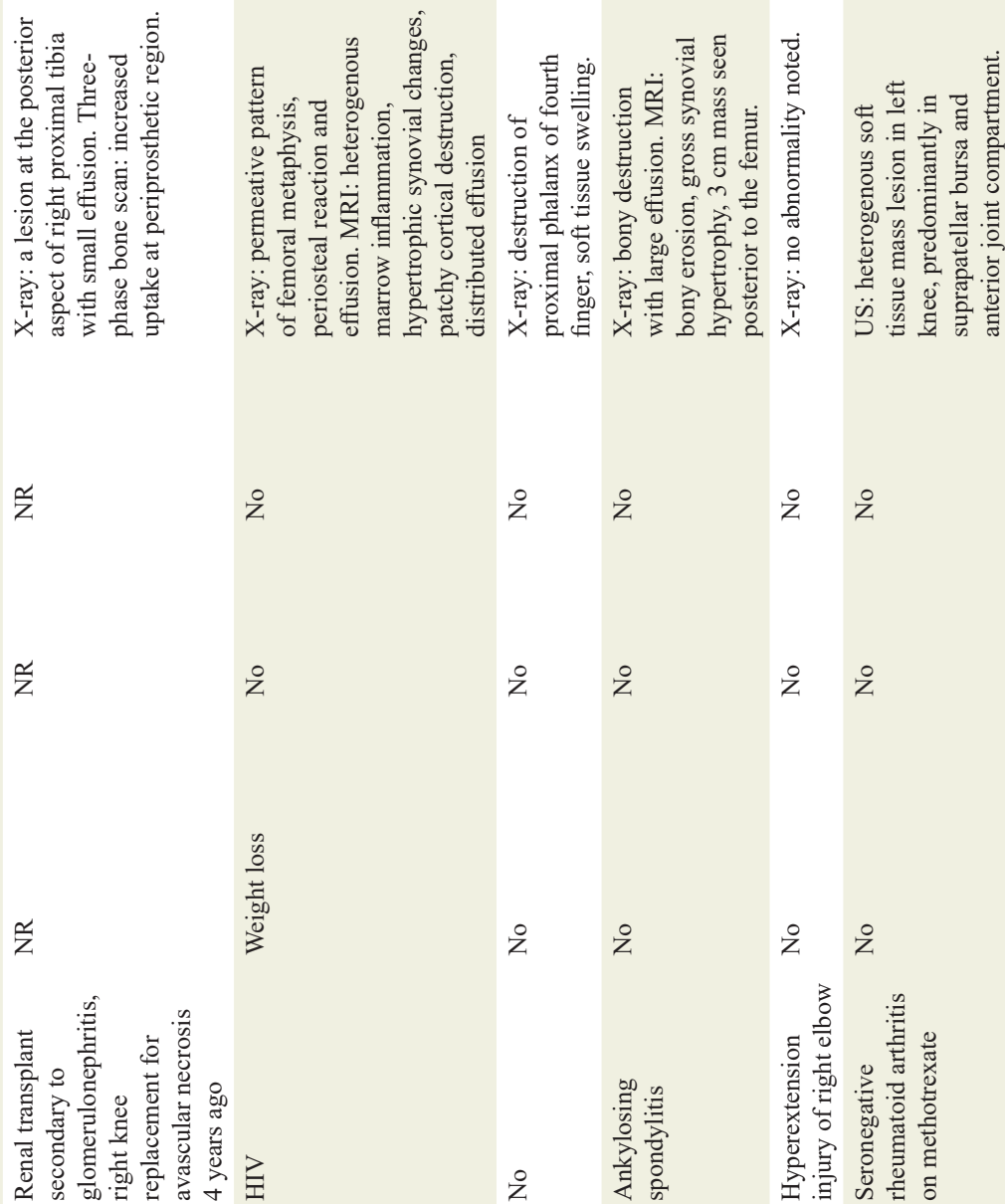

$\stackrel{\circ}{2}$

$\stackrel{\circ}{z}$

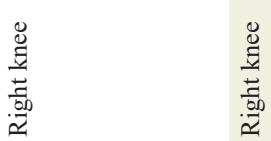

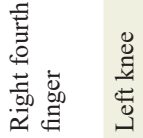

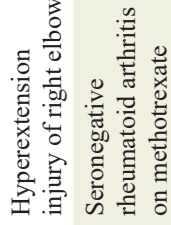

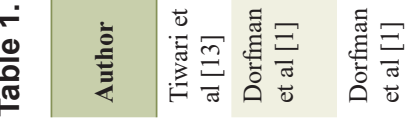

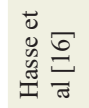

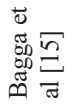

离离

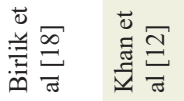

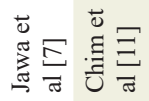




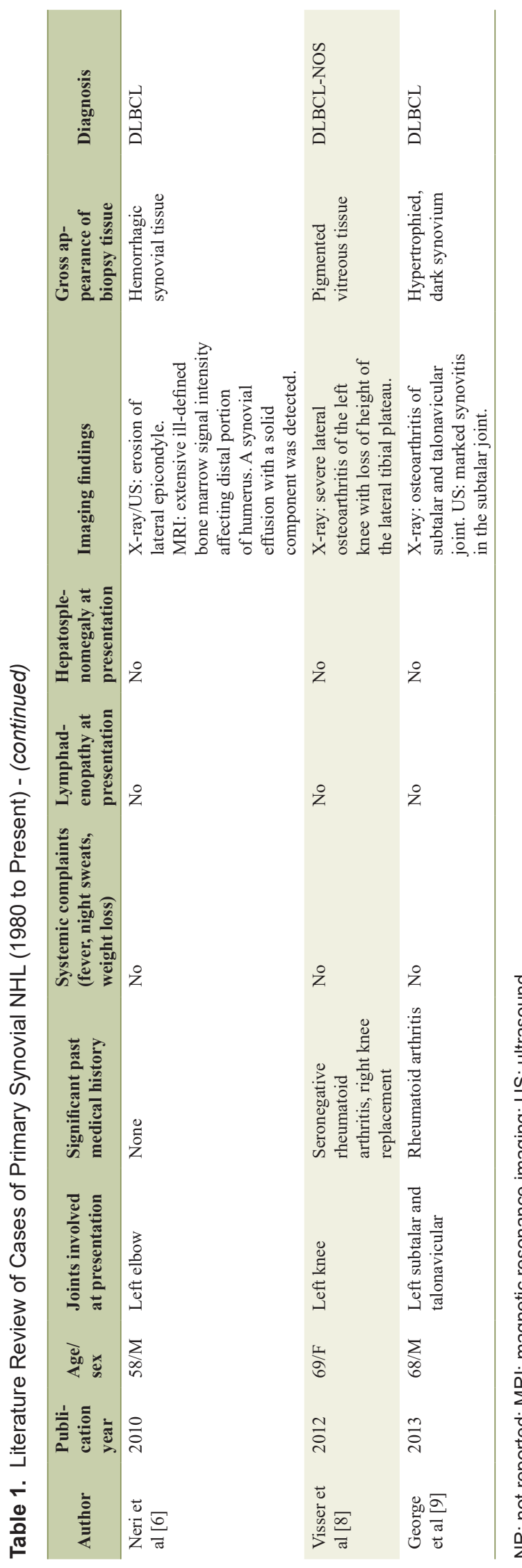

of extranodal involvement is the gastrointestinal tract.

Primary bone lymphoma is a rare disease and it accounts for 3-5\% of all extranodal NHLs [5]. Synovial involvement can occur due to direct extension from bone. Primary synovium lymphoma without bone involvement is extremely rare. On the review of the English-language literature from 1980 to present, we found 14 cases of primary synovial B-cell NHL [1, 6-18] (Table 1). The reported literature showed various joint involvement such as knee (most common), elbow and small joints of hand and feet. The presenting symptoms primarily included joint pain, stiffness, swelling, or reduced mobility. Three patients had associated systemic complaints such as fever, night sweats or weight loss. Only one patient had lymphadenopathy (left inguinal). No patient had hepatosplenomegaly on presentation. Studies have shown increased risk of NHL specifically DLBCL in patients with rheumatoid arthritis and immunocompromised states $[19,20]$.

Our patient had a history of bilateral knee osteoarthritis. Our patient presented as a clinically challenging diagnostic problem due to several reasons. 1) Due to rarity of primary synovial DLBCL and a recent uneventful right total knee replacement, the clinical suspicion for the disease was very low. 2) The patient did not show any systemic symptoms of DLBCL such as fever, weight loss or night sweats or signs such as lymphadenopathy or hepatosplenomegaly. 3) The patient's worsening symptoms of left knee pain, swelling and reduced mobility were attributed to progressing osteoarthritis. 4) Intraoperative findings of marked synovial hypertrophy and areas of fibrotic tissue were likely attributed to osteoarthritis versus infection. Therefore, the tissue samples were sent for cultures and not for histology. These factors may have caused delay in the diagnosis by as much as 14 weeks. On subsequent revision procedure, the surgeon noticed areas of necrosis and dark colored masses and samples were sent for histological analysis.

It is prudent to consider B-cell NHL as a differential diagnosis in a patient with unremitting joint pain, swelling and reduced mobility after a joint replacement for osteoarthritis. In our clinical practice, we do not routinely send tissue samples for histological analysis during elective joint arthroplasties. However, intraoperatively any abnormal appearing synovium (such as marked hypertrophy in our case), bone or intra-articular tissue must be sent for histological analysis for timely diagnosis. Tissue biopsy can lead to definitive diagnosis.

\section{Conclusion}

Our report illustrates a very rare case of a 74-year-old woman diagnosed with primary synovial DLBCL presenting as mechanical loosening of internal knee prosthetic joint. It is crucial to be aware of atypical presentation of DLBCL because it can lead to timely diagnosis and prompt treatment of this life-threatening yet a curable condition.

\section{Acknowledgments}

None to declare. 


\section{Financial Disclosure}

None to declare.

\section{Conflict of Interest}

Each author declares that there is no conflict of interest regarding the publication of this paper.

\section{Informed Consent}

Informed consent was given by the patient.

\section{Author Contributions}

KA and NA drafted the manuscript and did literature review. ML contributed to the pathological section of the manuscript.

\section{References}

1. Dorfman HD, Siegel HL, Perry MC, Oxenhandler R. NonHodgkin's lymphoma of the synovium simulating rheumatoid arthritis. Arthritis Rheum. 1987;30(2):155-161.

2. Morton LM, Wang SS, Devesa SS, Hartge P, Weisenburger DD, Linet MS. Lymphoma incidence patterns by WHO subtype in the United States, 1992-2001. Blood. 2006;107(1):265-276.

3. A clinical evaluation of the International Lymphoma Study Group classification of non-Hodgkin's lymphoma. The Non-Hodgkin's Lymphoma Classification Project. Blood. 1997;89(11):3909-3918.

4. Moller MB, Pedersen NT, Christensen BE. Diffuse large B-cell lymphoma: clinical implications of extranodal versus nodal presentation - a population-based study of 1575 cases. Br J Haematol. 2004;124(2):151-159.

5. Freeman C, Berg JW, Cutler SJ. Occurrence and prognosis of extranodal lymphomas. Cancer. 1972;29(1):252260.

6. Neri R, Giorgetti M, Zampa V, Iacopetti V, Vannucci P, Tognetti A, Bombardieri S. Case report. Elbow monoarthritis revealing B cell non-Hodgkin's lymphoma. Clin Rheumatol. 2013;(Suppl 1):S111-114.

7. Jawa A, Lieberman AE, Alexieva CC, Jupiter JB. Primary intra-articular non-Hodgkin's lymphoma of the elbow. A case report. J Bone Joint Surg Am. 2006;88(12):27302734.

8. Visser J, Busch VJ, de Kievit-van der Heijden IM, ten Ham AM. Non-Hodgkin's lymphoma of the synovium discovered in total knee arthroplasty: a case report. BMC Res Notes. 2012;5:449.

9. George DA, Afsharpad A, Chifu MD, Ray P. Large B cell lymphoma of the subtalar and talonavicular joint synovium. BMJ Case Rep. 2013;2013.

10. Cosatti MA, Pisoni CN, Altuve JL, Lorente C. B Cell Lymphoma mimicking Rheumatoid Arthritis. Rev Fac Cien Med Univ Nac Cordoba. 2016;73(1):46-49.

11. Chim CS, Pang YY, Ooi GC, Mok MY, Shek TW. EBV-associated synovial lymphoma in a chronically inflamed joint in rheumatoid arthritis receiving prolonged methotrexate treatment. Haematologica. 2006;91(8 Suppl):ECR31

12. Khan SY, Hutchinson DG. Primary synovial non-Hodgkin's lymphoma in association with ankylosing spondylitis. Rheumatology (Oxford). 2004;43(3):391.

13. Tiwari I, Thomas DR, Pathy MS, Clement DA. NonHodgkin's lymphoma presenting in synovial membrane. Br J Clin Pract. 1982;36(9):327-328.

14. Peeva E, Davidson A, Keiser HD. Synovial non-Hodgkin's lymphoma in a human immunodeficiency virus infected patient. J Rheumatol. 1999;26(3):696-698.

15. Bagga S, Gupta SM, Johns W. Scintigraphic detection of a disseminated synovial lymphoma in a postrenal transplant patient. Clin Nucl Med. 1996;21(8):615-618.

16. Haase KK, Durk H, Baumbach A, Saal J, Wehrmann M. Non-Hodgkin's lymphoma presenting as knee monoarthritis with a popliteal cyst. J Rheumatol. 1990;17(9):12521254.

17. Jamieson KA, Beggs I, Robb JE. Synovial presentation of non-Hodgkin's lymphoma. Br J Radiol. 1998;71(849):980-982.

18. Birlik M, Akar S, Onen F, Ozcan MA, Bacakoglu A, Ozkal S, Manisali M, et al. Articular, B-cell, non-Hodgkin's lymphoma mimicking rheumatoid arthritis: synovial involvement in a small hand joint. Rheumatol Int. 2004;24(3):169-172.

19. Baecklund E, Sundstrom C, Ekbom A, Catrina AI, Biberfeld P, Feltelius N, Klareskog L. Lymphoma subtypes in patients with rheumatoid arthritis: increased proportion of diffuse large B cell lymphoma. Arthritis Rheum. 2003;48(6):1543-1550.

20. Blinder V, Fisher SG, Lymphoma Research Foundation NY. The role of environmental factors in the etiology of lymphoma. Cancer Invest. 2008;26(3):306-316. 